\title{
Effects of side-dominance on knee joint proprioceptive target-matching asymmetries
}

\author{
K Galamb $^{1,2 *}$, B Szilágyi ${ }^{1 *}$, OM Magyar ${ }^{1}$, T Hortobágyi ${ }^{3}$, R Nagatomi ${ }^{4,5}$, M Váczi $^{6}$, \\ J Négyesi ${ }^{1,4}$ \\ ${ }^{1}$ Pain Clinic, Budapest, Hungary \\ ${ }^{2}$ Department of Movement, Human and Health Sciences, University of Rome, Rome, Italy \\ ${ }^{3}$ Center for Human Movement Sciences, University Medical Center Groningen, University of Groningen, \\ Groningen, The Netherlands \\ ${ }^{4}$ Department of Medicine and Science in Sports and Exercise, Tohoku University Graduate School of Medicine, \\ Sendai, Japan \\ ${ }^{5}$ Division of Biomedical Engineering for Health \& Welfare, Tohoku University Graduate School of Biomedical \\ Engineering, Sendai, Japan \\ ${ }^{6}$ Institute of Sport Sciences and Physical Education, University of Pécs, Pécs, Hungary
}

Received: November 8, 2017

Accepted: August 13, 2018

\begin{abstract}
Aims: Right- and left-side-dominant individuals reveal target-matching asymmetries between joints of the dominant and non-dominant upper limbs. However, it is unclear if such asymmetries are also present in lower limb's joints. We hypothesized that right-side-dominant participants perform knee joint target-matching tasks more accurately with their non-dominant leg compared to left-side-dominant participants. Methods: Participants performed position sense tasks using each leg by moving each limb separately and passively on an isokinetic dynamometer. Results: Sidedominance affected $(p<0.05)$ knee joint absolute position errors only in the non-dominant leg but not in the dominant leg: right-side-dominant participants produced less absolute position errors $\left(2.82^{\circ} \pm 0.72^{\circ}\right)$ with the non-dominant leg compared to left-side-dominant young participants $\left(3.54^{\circ} \pm 0.33^{\circ}\right)$. Conclusions: In conclusion, right-side-dominant participants tend to perform a target-matching task more accurately with the non-dominant leg compared to left-side-dominant participants. Our results extend the literature by showing that right-hemisphere specialization under proprioceptive target-matching tasks may be not evident at the lower limb joints.
\end{abstract}

Keywords: handedness, laterality, position sense, knee joint, dynamometer

\section{Introduction}

Due to the evolutionary specialization of the left hemisphere for skilled motor activities (12, $13,39), 90 \%$ of healthy adults are right-hand-dominant and perform fundamental manual motor tasks with the right hand $(27,33,44)$. This behavioral asymmetry is known as "righthandedness."

Proprioception is an essential element of motor control. Activation of muscle, skin, and joint receptors allows sensing the orientation of body and body parts even in the absence of vision [for reviews, see $(7,28)$ ]. Right-handed participants perform proprioceptive targetmatching tasks more accurately when using the non-dominant left thumb $(25,29)$, elbow

\footnotetext{
* These authors contributed equally to this work.

Corresponding author: János Négyesi

Department of Medicine and Science in Sports and Exercise, Tohoku University Graduate

School of Medicine, 2-1, Seiryo-machi, Aoba-ku, Sendai 980 8575, Japan

Phone/Fax: +81 22717 8588, E-mail: negyesi@dc.tohoku.ac.jp
} 
$(9,10,18)$, or multiple joints of the upper limb (ankle, knee, shoulder, and finger) (15) compared to left-handed participants performing the same task with the non-dominant right hand. One reason could be that kinesthesia is associated with a network of active brain areas (e.g., motor areas, cerebellum, and high-order somatosensory areas) in right-handed healthy participants, providing evidence for a right-hemisphere dominance for perception of limb movement (23). Although the non-preferred arm/hemisphere system is specialized for static limb position control, whereas the preferred arm/hemisphere system is responsible for dynamic limb trajectory control $(31,32)$, this asymmetry appears to be selective for right handers, but not for left handers (34). These data suggest that right-hemisphere specialization underlies proprioceptive feedback $(8,23)$. On the other hand, in a few cases, left-handed individuals also had smaller target-matching task errors when matching with the nondominant compared to the dominant arm (11), and some previous studies even failed to present target-matching asymmetry between upper limb joints on the right and left sides of the body. These contradictory results are probably due to the inconsistencies in experimental modalities $(2,24,29)$ or to the low number $(3-5)$ of testing trials $(1,2)$.

Most previous studies examined the effects of handedness on the proprioception of upper limb joints $(9,10,15,18,25,29)$, so it is unclear if right-hemisphere specialization for proprioceptive target-matching tasks $(8,23)$ is also evident in lower limb joints. The effects of footedness on leg proprioception have been poorly investigated, even though it might be a better indicator of brain lateralization (5), being less affected by external and societal factors than handedness (41). Although it has been shown that knee joint position sense is not more accurate in the non-preferred left limb under non-weight-bearing, partial weight-bearing, and full weight-bearing conditions (2), strongly right-side-dominant participants consistently sense movements more accurately using the left joints on both the upper and lower limbs (15).

Nevertheless, further work is needed to systematically determine whether proprioceptive asymmetry is evident in lower limb. Despite the large quantity of data on upper limb targetmatching behavior, it remains unknown whether lower limb proprioceptive asymmetry is different between right- and left-side-dominant individuals. Conferring with the data on upper limb proprioception, answering this question would provide a deeper insight into the mechanism of laterality. Therefore, the purpose of this study was to determine if side-dominance affects knee joint target-matching asymmetries between the dominant and non-dominant legs. We hypothesized that right-side-dominant participants perform knee joint target-matching tasks more accurately with their non-dominant leg compared to leftside-dominant participants.

\section{Materials and Methods}

\section{Participants}

Twenty-four healthy adults (age $=23.6 \pm 3.6$ years, range $=20-35,13$ men) volunteered for the experiment and were assigned to a right-side-dominant (RD; $n=12$ ) or a left-sidedominant (LD; $n=12$ ) group. To determine whether the participants were strongly right-sidedominant, both handedness and leg dominance were measured. Handedness was determined using the Edinburgh Handedness Inventory (26), a scale that is used to measure the degree of hand laterality in daily activities, such as writing, drawing, throwing, using scissors, brushing teeth, opening a box, striking a match, and using a pair of scissors, knife, spoon, and a broom. 
Leg dominance was determined by one- or two-foot item skill tests such as kicking a ball or stepping up on a chair (38). Laterality index was calculated by totaling the number of tasks performed using the right limb (R) and the number of tasks performed using the left limb (L) and computing the following statistic: $(\mathrm{R}-\mathrm{L}) /(\mathrm{R}+\mathrm{L}) \times 100$. Laterality index was $96 \pm 13$ for handedness and $99 \pm 2$ for footedness in right-side-dominant, and $-89 \pm 14$ and $-94 \pm 10$ in left-side-dominant participants, showing strong right- or left-side dominance, respectively. Participants had no past or current neurological or orthopedic disorders. After giving both verbal and written explanation of the experimental protocol, participants signed an informed consent in accordance with the Declaration of Helsinki.

\section{Procedures}

Sample size calculations [G*Power 3.1.7 (6)] for position error between right- and left-sidedominant participants were performed based on a previous study (35), which measured the accuracy and instability of arm position sense as a function of age, sex, and arm. Power analysis for one-way analysis of variance (ANOVA) was used to determine the sample size. High levels of interindividual variability are known to be associated with target-matching asymmetry between the dominant and non-dominant limbs that can make it difficult to detect changes in the measured variables, such as absolute position error. Therefore, only small or moderate effect size needs to be used for prestatistical power analysis, which results in a total sample size of 22, assuming type-I error of 0.05 and power of 0.80 . Twenty-four participants per group were recruited with consideration for dropouts.

Participants visited the laboratory once. The two legs were tested in a random order. Position sense measurements were performed on an isokinetic dynamometer (Cybex NORM, Cybex, Division of Lumex, Inc., Ronkonkoma, New York, USA). Figure 1 shows the

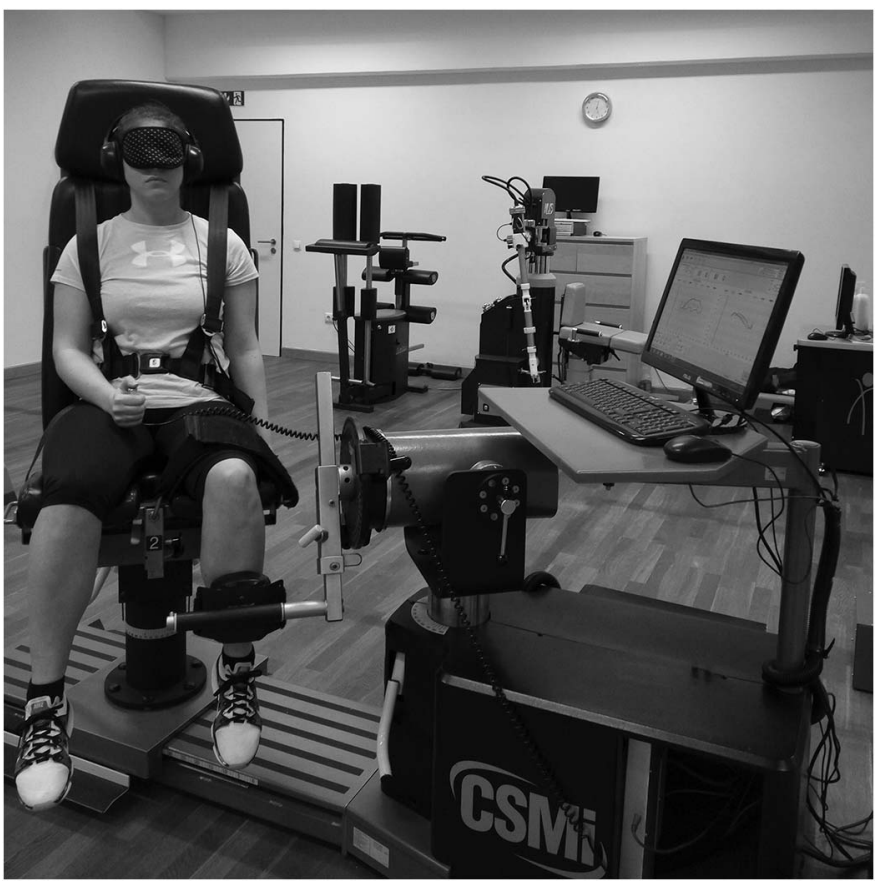

Fig. 1. Setup for the proprioception measurements. Participant was seated in the dynamometer chair in an upright position. One leg hung freely over the edge of the chair and the other leg was fixed to the attached freemoving arm, with a flexion angle of approximately $90^{\circ}$. Subject wore blindfolds for both tasks to eliminate vision and headphones with white noise in the motion sense task to eliminate auditory cues 
experimental setup. Participants wore a blindfold for both tasks to eliminate vision, and headphones with white noise in the motion sense task to eliminate auditory cues. Participants sat on the dynamometer seat in an upright position. One leg hung freely over the edge of the dynamometer seat and the other leg was attached to the dynamometer's lever.

The measurement of position sense was calculated based on a previous study (4). To perform position sense measurements without any input from active muscle contractions that could influence the participant's perception, measurements were performed by moving the limb passively. Before data collection, a test trial was performed to familiarize the participant with the procedure. Data were collected at targets of $30^{\circ}, 45^{\circ}$, and $60^{\circ}$ in a random order to minimize the potential influence of learning. The initial starting position was $90^{\circ}$ of knee flexion. Participants were instructed to focus on the position of the leg and then the leg was passively moved at a velocity of $4 \%$ s toward the target angle specified by the protocol. The target angle was then held for $5 \mathrm{~s}$ before the dynamometer's arm returned to the initial starting position $\left(90^{\circ}\right.$ of knee flexion). After $5 \mathrm{~s}$, the knee joint was passively extended again at a speed of $4 \%$, and the participant was instructed to push a hold button as soon as he or she sensed that the previously practiced target position had been reached. To maintain attentional alert, after every five trials, participants counted backward by seven, starting from a two-digit number given by the experimenter.

Each target angle was repeated five times, giving 15 data points both for the dominant and the non-dominant leg. Any deviation in degrees from the target position was defined as the absolute position error and used in the data analyses. The 15 data points were then averaged to calculate an average per participant for both the dominant and non-dominant legs.

\section{Statistical analyses}

We report the data as mean $\pm \mathrm{SD}$. All data were checked for normal distribution using the Shapiro-Wilk's test. Variables that were not normally distributed were log-transformed. The analyses were performed on the transformed data using SPSS (v. 22.0) but the data are reported in the non-transformed form. A $2 \times 2$ [side-dominance (right-side-dominant; leftside-dominant) $\times$ Leg (dominant; non-dominant)] ANOVA was used to measure, if handedness affects leg function defined as asymmetries in knee joint absolute position errors between the dominant and non-dominant legs. Complementary independent samples $t$-tests were used to detect the differences in absolute position errors between and within groups. In addition, effect sizes between and within groups were calculated as Cohen's $d$ (3). Finally, one-way ANOVAs in each group for both legs were performed to investigate, if absolute position errors differ between the three target angles $\left(30^{\circ}, 45^{\circ}\right.$, and $\left.60^{\circ}\right)$. Statistical significance was set at $p<0.05$. Results were interpreted by a confidence interval of $95 \%$.

\section{Results}

Table I shows the proprioceptive target-matching data for both legs. There were differences in proprioceptive target-matching asymmetries based on side-dominance $\left(F_{2,21}=7.819, p=\right.$ 0.003 , Wilk's $=0.573$, partial $\left.\eta^{2}=0.43\right)$. Side-dominance affected knee joint absolute position errors in the non-dominant leg $\left(F_{1,22}=12.398, p=0.002\right.$, partial $\left.\eta^{2}=0.36\right)$ but not in the dominant leg $\left(F_{1,22}=2.196, p=0.153\right.$, partial $\left.\eta^{2}=0.09\right)$. Subsequent $t$-tests showed that RD participants produced less $(p=0.002)$ absolute position errors with the non-dominant $\operatorname{leg}\left(2.82^{\circ} \pm 0.72^{\circ}\right)$ compared to participants in the LD group $\left(3.53^{\circ} \pm 0.32^{\circ}\right.$, Cohen's $\left.d=1.27\right)$ 
Table I. Mean absolute position errors obtained from a proprioceptive target-matching task in the dominant and nondominant legs

\begin{tabular}{|l|c|c|}
\hline \multirow{2}{*}{} & Dominant leg & Non-dominant leg \\
\cline { 2 - 3 } & Mean $( \pm$ SD) & Mean $( \pm$ SD) \\
\hline RD & $3.49(1.03)$ & $2.66(0.45)$ \\
\hline LD* $^{*}$ & $2.92(0.38)$ & $3.53(0.32)$ \\
\hline
\end{tabular}

Values are absolute position errors (degrees). SD: standard deviation; RD: participants with right-side dominance $(n=12)$; LD: participants with left-side dominance $(n=12)$.

* Significant difference between dominant and non-dominant legs $(p<0.05)$

A

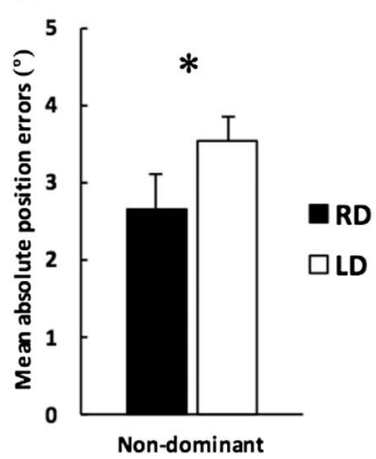

B

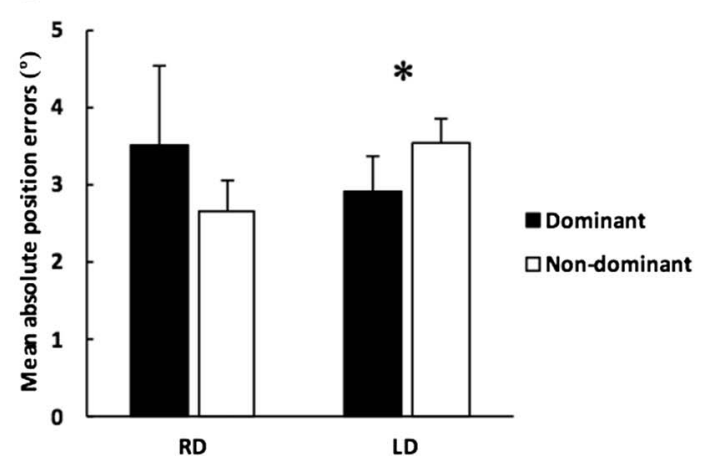

Fig. 2. Side-dominance influences knee joint proprioceptive target-matching asymmetries. (A) Right-side-dominant participants (RD; filled bar) produced less absolute errors during position target-matching test with the non-dominant leg compared to left-side-dominant participants (LD; open bar). (B) Left-side-dominant participants (LD) produced

less absolute mean errors with the left-dominant (filled bar) than the right non-dominant (open bar) leg

(Fig. 2A). Furthermore, LD group $(p=0.003$ ) produced less absolute position error with the left-dominant leg $\left(2.92^{\circ} \pm 0.38^{\circ}\right)$ compared to the right non-dominant $\left(3.53^{\circ} \pm 0.32^{\circ}\right.$, Cohen's $d=1.73$ ) leg (Fig. 2B). No significant interactions were found between the position target angles in the dominant and the non-dominant leg either in $\operatorname{RD}\left(F_{2,33}=0.015\right.$, $p=0.985 ; F_{2,33}=1.024, p=0.370$, respectively $)$, or in LD groups $\left(F_{2,33}=0.254, p=0.777\right.$; $F_{2,33}=0.216, p=0.807$, respectively).

\section{Discussion}

We determined the effects of side-dominance on target-matching asymmetry between the dominant and non-dominant legs. In contrast with previous studies $(9,10,18)$, which reported more accurate target-matching in the non-dominant joints compared to the dominant ones, the present results revealed no differences in accuracy between dominant and nondominant legs. On the other hand, we found that right-side-dominant participants were more accurate in the target-matching task with the non-dominant leg than the left-side-dominant subjects. 
To the best of our knowledge, this is the first study that determined whether targetmatching was more accurate when using the non-dominant leg, just as it was shown in thumb $(25,29)$, elbow $(9,10,18)$, or in multiple joints of the upper limb (ankles, knees, shoulders, and fingers) (15) in right-handed individuals and in elbow (11) in left-handed individuals. In contrast to our hypothesis, we found no asymmetry in the knee joint targetmatching task in right-side-dominant participants. In line with our results, several previous studies also failed to present target-matching asymmetry between upper limb joints on the right and left sides of the body $(2,24,29)$ probably due to the different methodologies. First, the lack of limb asymmetry observed in joint position reproduction tests may be due to the low number $(3-5)$ of testing trials $(1,2)$. Second, studies that aim to measure proprioception used target position generation or matching tests; however, these methods involve different mechanisms. Sensory feedback has important role in all aspects of movement and proprioception provides the primary source of sensory information. In passive movement control, input from cutaneous receptors seems to play a greater role in sensory feedback, as fusimotor activity and the sensory feedback from muscle spindles are diminished. In contrast, in active movement, both fusimotor drive and muscle spindle feedback are involved, thus playing a more dominant role (45). Furthermore, position sense tends to be better for the more proximal than the distal joints (14), reflecting differences in the number of muscle spindles present in the joints (36). It has been argued that proprioceptive asymmetry may be evident only at distal joints and not at proximal joints due to the specific organization of the motor system. While proximal musculature is innervated by both hemispheres, more distal musculature has been thought to be innervated largely by the contralateral hemisphere $(19,22)$.

On the other hand, right-side-dominant participants produced less absolute position errors $\left(2.82^{\circ} \pm 0.72^{\circ}\right)$ with the non-dominant leg compared to left-side-dominant young participants $\left(3.54^{\circ} \pm 0.33^{\circ}\right)$, suggesting that the non-dominant arm/contralateral hemisphere specialization for the utilization of proprioceptive feedback $(8,23)$ seems to be selective only for right handers, but not for left handers (34). In right-handed healthy participants, kinesthesia is associated with a network of active brain areas including motor areas, the cerebellum, and the right fronto-parietal areas including high-order somatosensory areas, providing evidence for a right-hemisphere dominance for perception of limb movement (23). The results from previous studies are controversial whether handedness is related to activation asymmetries in different parts of the brain. For example, there is a strong relationship between handedness and activation asymmetries in the motor $(42,43)$ and somatosensory cortex (20); others found that motor cortex asymmetry was less pronounced in left handers than the right ones $(17,37)$ and the size of hand sensory representation from thumb to little finger was similar in the two hemispheres (40). Although weaker lateralization in left-handed than in right-handed individuals is often suggested, reversed asymmetries were also reported for the left-handed population (11). The nature of side-dominance, including handedness, is a consequence of brain lateralization through complex motor control processes [for reviews, see $(16,30)$ ]. Left handedness is a marker of atypical cerebral lateralization; therefore, left-handed individuals have cognitive functions distributed more evenly across the left and right cerebral hemispheres. This can be one of the reasons why left-handed individuals are less likely to exhibit the functional asymmetries seen in right-handed individuals. Second, right-handed individuals have lower left-hand thresholds than the right; however, the asymmetry is based on cerebral lateralization. Therefore, left-handed participants may not exhibit the same central and peripheral asymmetry (21). 
A limitation of this study is the small effect size. While side-dominance showed a large effect on knee joint absolute position errors in the non-dominant leg (Cohen's $d=1.27$ ), the difference between right- and left-side-dominant subjects was only $0.7^{\circ}$. Although prestudy statistical power calculation revealed that 22 subjects would be optimal to detect differences in position error between groups, increasing the sample size would probably have a positive influence on detecting more remarkable differences.

In summary, our results suggest that right-side-dominant participants tend to perform a target-matching task with a greater accuracy with the non-dominant leg than leftside-dominant individuals. Our results extend the literature by showing that right-hemisphere specialization under proprioceptive target-matching tasks may be not evident in the knee joints. Future studies need to recruit subjects with ambidexterity (subjects equally using both the left and the right hands/legs) or "crossed laterality" (subjects with right-hand-left-leg dominance or left-hand-right-leg dominance) to reliably determine the relationships between handedness and footedness and its influence on joint proprioception. Future researches should also be initiated to determine whether age influences differently knee joint targetmatching asymmetries between right- and left-side-dominant individuals. Finally, we strongly encourage researchers to perform neuroanatomical studies to evaluate the underlying physiological mechanisms for both upper and lower limb joint position sense through aging that would further be informative for physiotherapists and trainers, who wish to maintain balance function in old age.

\section{Conflict of interest}

All authors declare no conflict of interest.

\section{Abbreviations}

ANOVA : analysis of variance

LD : participants with left-side dominance

RD : participants with right-side dominance

\section{REFERENCES}

1. Adamo DE, Alexander NB, Brown SH: The influence of age and physical activity on upper limb proprioceptive ability. J. Aging Phys. Act. 17, 272-293 (2009)

2. Bullock-Saxton JE, Wong WJ, Hogan N: The influence of age on weight-bearing joint reposition sense of the knee. Exp. Brain Res. 136, 400-406 (2001)

3. Cohen J (1988): Statistical power analysis for the behavioral sciences (2nd ed.). Lawrence Erlbaum Associates, Hillsdale, NJ

4. Dieling S, van der Esch M, Janssen TW: Knee joint proprioception in ballet dancers and non-dancers. J. Dance Med. Sci. 18, 143-148 (2014)

5. Elias LJ, Bryden MP: Footedness is a better predictor of language lateralisation than handedness. Laterality 3, 41-51 (1998)

6. Faul F, Erdfelder E, Lang AG, Buchner A: G*Power 3: a flexible statistical power analysis program for the social, behavioral, and biomedical sciences. Behav. Res. Methods 39, 175-191 (2007)

7. Gandevia SC, Refshauge KM, Collins DF: Proprioception: peripheral inputs and perceptual interactions. Adv. Exp. Med. Biol. 508, 61-68 (2002)

8. Goble DJ, Brown SH: Task-dependent asymmetries in the utilization of proprioceptive feedback for goaldirected movement. Exp. Brain Res. 180, 693-704 (2007)

9. Goble DJ, Brown SH: Upper limb asymmetries in the matching of proprioceptive versus visual targets. J. Neurophysiol. 99, 3063-3074 (2008) 
10. Goble DJ, Lewis CA, Brown SH: Upper limb asymmetries in the utilization of proprioceptive feedback. Exp. Brain Res. 168, 307-311 (2006)

11. Goble DJ, Noble BC, Brown SH: Proprioceptive target matching asymmetries in left-handed individuals. Exp. Brain Res. 197, 403-408 (2009)

12. Gonzalez CL, Goodale MA: Hand preference for precision grasping predicts language lateralization. Neuropsychologia 47, 3182-3189 (2009)

13. Goodale MA: Hemispheric differences in motor control. Behav. Brain Res. 30, $203-214$ (1988)

14. Hall LA, McCloskey DI: Detections of movements imposed on finger, elbow and shoulder joints. J. Physiol. 335, 519-533 (1983)

15. Han J, Anson J, Waddington G, Adams R: Proprioceptive performance of bilateral upper and lower limb joints: side-general and site-specific effects. Exp. Brain Res. 226, 313-323 (2013)

16. Hatta T: Handedness and the brain: a review of brain-imaging techniques. Magn. Reson. Med. Sci. 6, 99-112 (2007)

17. Jung P, Baumgärtner U, Bauermann T, Magerl W, Gawehn J, Stoeter P, Treede RD: Asymmetry in the human primary somatosensory cortex and handedness. Neuroimage 19, 913-923 (2003)

18. Kurian G, Sharma NK, Santhakumari K: Left-arm dominance in active positioning. Percept. Mot. Skills 68, 1312-1314 (1989)

19. Kuypers HG: A new look at the organization of the motor system. Prog. Brain Res. 57, 381-403 (1982)

20. Legon W, Dionne JK, Meehan SK, Staines WR: Non-dominant hand movement facilitates the frontal N30 somatosensory evoked potential. BMC Neurosci. 11, 112 (2010)

21. Meador KJ, Ray PG, Day L, Ghelani H, Loring DW: Physiology of somatosensory perception: cerebral lateralization and extinction. Neurology 51, 721-727 (1998)

22. Müller F, Kunesch E, Binkofski F, Freund HJ: Residual sensorimotor functions in a patient after right-sided hemispherectomy. Neuropsychologia 29, 125-145 (1991)

23. Naito E, Roland PE, Grefkes C, Choi HJ, Eickhoff S, Geyer S, Zilles K, Ehrsson HH: Dominance of the right hemisphere and role of area 2 in human kinesthesia. J. Neurophysiol. 93, 1020-1034 (2005)

24. Naughton J, Adams R, Maher C: Discriminating overhead points of contact after arm raising. Percept. Mot. Skills 95, 1187-1195 (2002)

25. Nishizawa S: Different pattern of hemisphere specialization between identical kinesthetic spatial and weight discrimination tasks. Neuropsychologia 29, 305-312 (1991)

26. Oldfield RC: The assessment and analysis of handedness: the Edinburgh Inventory. Neuropsychologia 9 , 97-113 (1971)

27. Perelle IB, Ehrman L: On the other hand. Behav. Genet. 35, 343-350 (2005)

28. Proske U, Gandevia SC: The proprioceptive senses: their roles in signaling body shape, body position and movement, and muscle force. Physiol. Rev. 92, 1651-1697 (2012)

29. Roy EA, MacKenzie C: Handedness effects in kinesthetic spatial location judgements. Cortex 14, 250-258 (1978)

30. Sainburg RL: Convergent models of handedness and brain lateralization. Front. Psychol. 5, 1092 (2014)

31. Sainburg RL: Evidence for a dynamic-dominance hypothesis of handedness. Exp. Brain Res. 142, 241-258 (2002)

32. Sainburg RL: Handedness: Differential specializations for control of trajectory and position. Exerc. Sport Sci. Rev. 33, 206-213 (2005)

33. Sartarelli M: Handedness, earnings, ability and personality. Evidence from the lab. PLoS One 11, e0164412 (2016)

34. Schmidt L, Artinger F, Stumpf O, Kerkhoff G: Differential effects of galvanic vestibular stimulation on arm position sense in right- vs. left-handers. Neuropsychologia 51, 893-899 (2013)

35. Schmidt L, Depper L, Kerkhoff G: Effects of age, sex and arm on the precision of arm position sense-left-arm superiority in healthy right-handers. Front. Hum. Neurosci. 7, 915 (2013)

36. Scott SH, Loeb GE: The computation of position sense from spindles in mono- and multiarticular muscles. J. Neurosci. 14, 7529-7540 (1994)

37. Sörös P, Knecht S, Imai T, Gürtler S, Lütkenhöner B, Ringelstein EB, Henningsen H: Cortical asymmetries of the human somatosensory hand representation in right- and left-handers. Neurosci. Lett. 271, 89-92 (1999)

38. Spry S, Zebas C, Visser M (1993): What is leg dominance? In: 11 International Symposium on Biomechanics in Sports ISBS - Conference Proceedings Archive, eds Hamill J, Derrick TR, Elliott EH, pp. 165-168. Available at: https://ojs.ub.uni-konstanz.de/cpa/article/view/1700 
39. Stone KD, Bryant DC, Gonzalez CL: Hand use for grasping in a bimanual task: evidence for different roles? Exp. Brain Res. 224, 455-467 (2013)

40. Tecchio F, Rossini PM, Pizzella V, Cassetta E, Romani GL: Spatial properties and interhemispheric differences of the sensory hand cortical representation: a neuromagnetic study. Brain Res. 767, 100-108 (1997)

41. Tran US, Stieger S, Voracek M: Evidence for general right-, mixed-, and left-sidedness in self-reported handedness, footedness, eyedness, and earedness, and a primacy of footedness in a large-sample latent variable analysis. Neuropsychologia 62, 220-232 (2014)

42. Triggs WJ, Calvanio R, Levine M: Transcranial magnetic stimulation reveals a hemispheric asymmetry correlate of intermanual differences in motor performance. Neuropsychologia 35, 1355-1363 (1997)

43. Volkmann J, Schnitzler A, Witte OW, Freund H: Handedness and asymmetry of hand representation in human motor cortex. J. Neurophysiol. 79, 2149-2154 (1998)

44. Vuoksimaa E, Koskenvuo M, Rose RJ, Kaprio J: Origins of handedness: a nationwide study of 30,161 adults. Neuropsychologia 47, 1294-1301 (2009)

45. Zazulak BT, Hewett TE, Reeves NP, Goldberg B, Cholewicki J: The effects of core proprioception on knee injury: a prospective biomechanical-epidemiological study. Am. J. Sports Med. 35, 368-373 (2007) 